\title{
A Brazilian initiative towards Digital Literacy: assessing Motivational Factors of e-Proinfo using TAM
}

\author{
Élton C. Marinhoo ${ }^{1,2}$, Mônica F. da Silva ${ }^{3}$, Éber Schmitz ${ }^{1,3}$, Antônio J. de Alencar ${ }^{1,3}$ \\ ${ }^{1}$ Programa de pós-graduação em Informática - PPGI/UFRJ \\ ${ }^{2}$ Decania do Centro de Ciências Matemáticas e da Natureza - CCMN/UFRJ \\ ${ }^{3}$ Instituto Tércio Pacitti de Aplicações e Pesquisas Computacionais - NCE/UFRJ \\ elton@ufrj.br, \{monica, eber\}@nce.ufrj.br, juarezalencar@br.inter.net
}

\begin{abstract}
This research aims to verify the impact of motivational factors in the use of a virtual learning environment, specifically; the factors perceived enjoyment, perceived usefulness and perceived ease-of-use. The environment studied was an initiative of Digital Literacy organized by Brazilian Ministry of Education: e-Proinfo, which is offered to elementary school students. In this multi-method research, a case study was applied in a private school at Rio de Janeiro, followed by an experiment with 20 students of this school. Qualitative data collected from the case study allowed us to endorse some of the experiment results on the Quantitative Phase. Previous researches indicate that teenagers tend to be motivated by playful environments, pointing to the importance of perceived enjoyment factor. Nevertheless, in this experiment, it was observed that perceived usefulness was the motivational factor with greatest impact on intention of use.
\end{abstract}

RESUMO. Esta pesquisa teve como objetivo verificar os impactos de fatores motivacionais no uso de um ambiente virtual de aprendizagem, especificamente, os fatores prazer percebido, utilidade percebida e facilidade percebida. $O$ ambiente estudado foi uma iniciativa de Alfabetização Digital organizada pelo Ministério da Educação: e-Proinfo, o qual é oferecido aos alunos do ensino médio. Nessa pesquisa multimétodo, foi feito um estudo de caso em uma instituição privada da zona oeste do Rio de Janeiro seguido de um experimento com 20 alunos dessa escola. A coleta de dados qualitativos do estudo de caso permitiu confirmar alguns resultados do experimento. Apesar de pesquisas anteriores indicarem que indivíduos na faixa etária pesquisada, adolescentes, tendem a se motivar com ambientes mais lúdicos, apontando para a importância do fator motivacional prazer percebido; nesse experimento, foi observado que o fator motivacional com maior impacto na intenção de uso do ambiente foi a utilidade percebida. 


\section{PALAVRAS CHAVE}

Ambiente Virtual de Aprendizagem (AVA), Technology Acceptance Model (TAM), Alfabetização Digital, Ensino Médio, e-Proinfo.

\section{INTRODUÇÃO}

A adoção de uma tecnologia tem sido tema de inúmeras pesquisas [Davis 1986; Dias 2001; Silva 2006], principalmente por seu papel estratégico em vários cenários. Essa investigação foi motivada pela necessidade de entender o comportamento de alunos do Ensino Médio e sua relação com a plataforma de ensino a distância e-Proinfo [MEC 2013] oferecida pelo MEC (Ministério da Educação) aos alunos do $1^{\circ}$ e $2^{\circ}$ anos do Ensino Médio. A investigação teve como ponto de partida a seguinte questão: Como os fatores motivacionais impactam no uso de um Ambiente Virtual de Aprendizagem (AVA) por alunos de uma instituição de ensino privada?

Assim, essa investigação científica versa sobre a aceitação de um AVA (o e-Proinfo) sob a perspectiva do modelo TAM (Technology Acceptance Model) [Davis 1986], e teve como foco três importantes fatores motivacionais para o uso de uma tecnologia com base em pesquisas anteriores [Davis 1986, 1989] [Dias 2001, 2002]: o prazer percebido, a utilidade percebida e a facilidade percebida. $\mathrm{O}$ objetivo do trabalho foi investigar como são percebidos esses fatores pelos alunos do Ensino Médio de uma instituição privada de Ensino com relação ao e-Proinfo, e como esses fatores impactam na intenção de uso desse ambiente.

\section{PROJETO E MODELO TEÓRICO DA PESQUISA}

Essa pesquisa pode ser classificada como uma pesquisa multimétodo, sendo composta por um estudo de caso e um experimento a fim de estudar o fenômeno da adoção de tecnologia a partir de visões diferentes e complementares. Essa pesquisa segue um modelo multimétodo transformativo [Creswell 2010], pois ocorre a coleta de dois tipos de dados obedecendo a diferentes tópicos de interesse. Nesse caso, há uma etapa qualitativa (um estudo de caso com foco na perspectiva da instituição de ensino) e uma etapa quantitativa (um experimento com foco na perspectiva do aluno), a fim de comparar diferentes percepções sobre o mesmo fenômeno (Fig. 1).

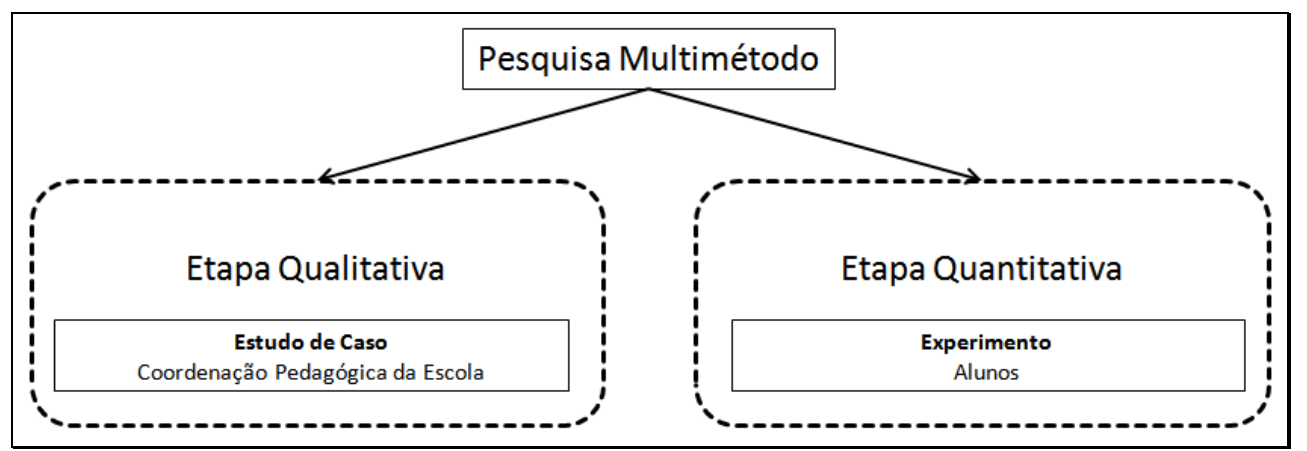

Fig. 1 - Projeto de Pesquisa multimétodo transformativa

O Estudo de Caso [Dias e Silva 2010] foi elaborado a partir de entrevistas com o coordenador pedagógico do Ensino Médio da instituição privada estudada e de visitas às 
dependências com questionamentos objetivos com alguns funcionários e acesso a documentos que descrevem o método de ensino da escola.

Já o experimento [Dias e Silva 2010] foi feito com 20 alunos do $1^{\circ}$ e $2^{\circ}$ anos do Ensino Médio dessa mesma instituição focando os fatores em estudo e com algumas perguntas abertas com o intuito de confirmar os fatores medidos. Os fatores em estudo seguem o modelo teórico exposto na Fig. 2. O uso real não pode ser avaliado, já que o AVA foi disponibilizado para este grupo apenas durante o experimento.

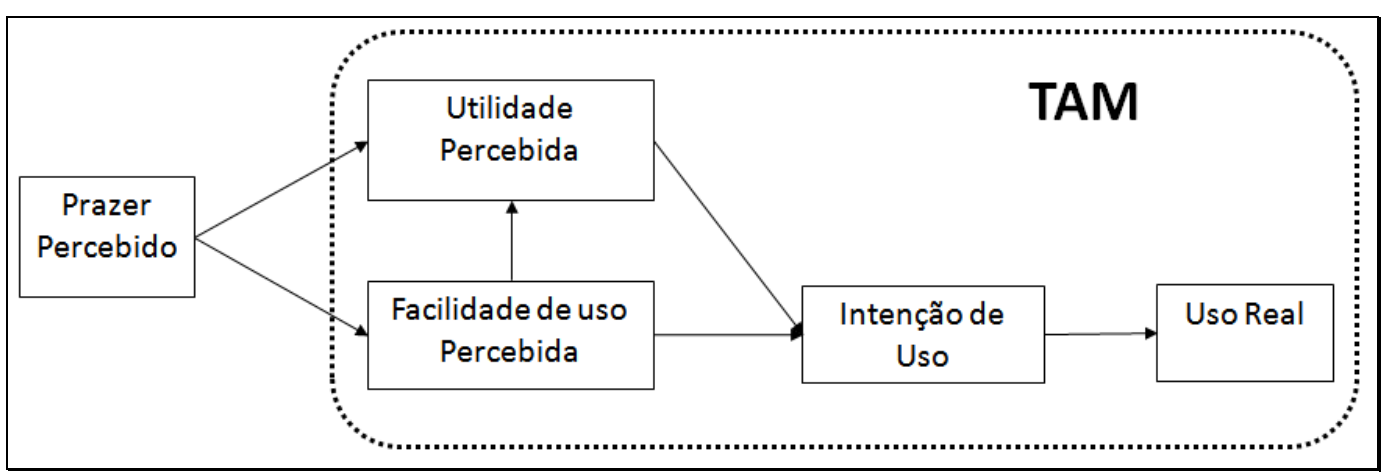

Fig. 2 - Modelo de Aceitação de Tecnologia - TAM

\section{AMBIENTE VIRTUAL DE APRENDIZAGEM (AVA)}

Um Ambiente Virtual de Aprendizagem (AVA) é, concretamente, um software, disponibilizado via Internet, onde podem ser montados cursos com as mais diversas opções de tecnologia. Um AVA é elaborado a fim de auxiliar no gerenciamento de conteúdos didáticos e possibilita a administração do curso e o acompanhamento constante do progresso dos participantes. Pode ser um complemento para aulas presenciais ou conter todo o material didático e avaliações do curso em questão.

Algumas opções de tecnologia oferecidas por intermédio de um AVA para interação entre os participantes são: fórum, bate-papo, mensagem de texto, vídeo, agenda, atividade objetiva entre outras. Dentre os tipos de participantes, um AVA pode ser usado por: alunos, professores, coordenadores e/ou outros atores que participem do processo.

\section{ADOÇÃO DE TECNOLOGIA}

O modelo TAM (Technology Acceptance Model) é um aprofundamento nos componentes atitudinais de pesquisas anteriores [Davis 1986] [Fishbein 1975], sendo esses componentes atitudinais formados a partir de dois fatores básicos: a utilidade percebida e a facilidade percebida. O prazer percebido foi escolhido como antecedente nessa pesquisa porque pesquisas anteriores [Dias 2001] [Silva 2006] indicam que indivíduos da faixa etária pesquisada percebem o ambiente computacional de forma mais lúdica, aliado à voluntariedade no uso do AVA.

O fator utilidade percebida de um Ambiente Virtual de Aprendizagem (AVA) foi definido para esse estudo como "o grau em que um aluno acredita que a utilização de um AVA aumentaria o seu desempenho na escola". Foram considerados os seguintes aspectos para capturar essa percepção de utilidade dos alunos: o desempenho alcançado, a melhoria da produtividade, a qualidade das tarefas e a utilidade do AVA em geral. 
O fator facilidade percebida de um AVA foi definido como "o grau em que um aluno acredita que a utilização de um AVA reduziria seu esforço físico elou mental", e essa percepção foi capturada através dos seguintes aspectos: facilidade no uso do AVA por ele mesmo, facilidade no uso do AVA para aprender e a facilidade do uso do AVA em geral.

O fator prazer percebido foi definido como "o grau em que um aluno acredita ser prazeroso utilizar um AVA, independente de qualquer outro fator", e esta percepção foi capturada através dos aspectos: excitação, envolvimento, diversão e prazer no uso do AVA.

\section{E-GOVERNMENT E ALFABETIZAÇÃO DIGITAL}

E-government é o uso das tecnologias da informação a fim de gerar produtos e serviços que se transformem em melhorias para a população em geral, tudo isso visando aproximar o governo do cidadão, superando os obstáculos de comunicação entre estas duas esferas. Inúmeras ferramentas podem ser utilizadas para atingir este objetivo, tais como portais de internet e aplicativos para telefonia móvel.

E-government pode ser entendido como a prestação de serviço público por meio eletrônico, utilizando os recursos de tecnologia da informação e comunicação em caráter remoto e disponível vinte e quatro horas por dia, sete dias por semana. Um dos serviços que o governo brasileiro vem prestando aos cidadãos é promover a inclusão digital por meio da alfabetização digital.

Para que a inclusão digital possa acontecer é necessário que o cidadão possua um dispositivo para conexão (um computador, um tablet ou mesmo um telefone celular) e acesso à internet. Contudo, isso não é o bastante para que o uso das tecnologias digitais ocorra.

Alfabetização Digital é a capacitação no acesso à informação na Internet, possibilitando a inclusão digital. Vale realçar que o termo "alfabetização digital", assim como o termo "alfabetização", encerra apenas o aprendizado dos códigos para que uma competência posterior se forme, a saber, a de construir uma argumentação ou construir sentido a partir do código aprendido, sendo este formado por palavras, elementos sonoros, itens gráficos, etc. [Buzato 2003].

A alfabetização digital assume papel de destaque na era digital uma vez que permite o aprendizado e o uso efetivo dos dispositivos digitais a fim de possibilitar o acesso aos conhecimentos disponibilizados nessas novas tecnologias.

Várias iniciativas vêm sendo conduzidas a fim de capacitar a sociedade no uso das novas tecnologias. Uma das iniciativas do Ministério da Educação foi a construção de um ambiente virtual de aprendizagem: e-Proinfo.

\section{DESCRIÇÃO DO CASO ESTUDADO E DO EXPERIMENTO}

O e-Proinfo é um ambiente virtual colaborativo de aprendizagem que permite a criação, administração e desenvolvimento de diversos tipos de eventos, tais como, cursos a distância, apoio a cursos presenciais, projetos de pesquisa e diversas outras formas de apoio a distância e ao processo ensino-aprendizagem. 
O e-Proinfo oferecido pelo Ministério da Educação [MEC 2013], possui um evento (um curso) com o conteúdo voltado para Qualificação em Tecnologia Digital. Este conteúdo tem como objetivo dar condições aos alunos de escolas da rede pública de apoiar a utilização dos laboratórios de informática de suas escolas iniciando-os ao uso e compreensão dos recursos de informática, democratizando o acesso as tecnologias de informação. Tópicos como Educação a Distância, Sociedade em rede e Evolução da Informática fazem parte do primeiro módulo do curso que é composto por quatro módulos sendo os demais sobre Hardware, Sistemas Operacionais e Manutenção de Computadores. Como opções tecnológicas disponíveis estão presentes fóruns, bate-papo, produção de texto, produção de texto coletivo e mural de recados, entre outros.

A instituição privada objeto desta pesquisa é uma instituição tradicional da zona oeste do Rio de Janeiro com 37 anos no setor de ensino. Iniciou suas atividades contemplando o Ensino Médio e o segundo segmento do Ensino Fundamental, e tinha aproximadamente 300 alunos. Hoje atua em todo o Ensino Médio e Fundamental, contando com mais de 2.000 (dois mil) alunos. O Coordenador Pedagógico entrevistado trabalha nessa instituição desde sua criação.

As aulas são ministradas no formato tradicional, auxiliadas por variados aparatos tecnológicos, possuindo inclusive uma unidade móvel de multimídia. A escola dispõe de um laboratório de informática com 25 computadores ligados a internet, o qual foi utilizado para viabilizar o experimento objeto dessa pesquisa.

A fim de melhor conhecer o perfil do aluno participante do experimento, foram coletadas informações demográficas sobre idade, sexo e série de cada indivíduo.

Os participantes, todos voluntários e sem conhecimento prévio do ambiente em estudo, foram dispostos no laboratório de informática durante o intervalo das aulas, que dura aproximadamente 30 minutos. Nos computadores do laboratório, em tela previamente preparada, o AVA proposto estava aberto para acessarem e, após as explicações iniciais sobre o objetivo do experimento, os alunos foram solicitados a efetuar 5 (cinco) tarefas com o intuito de estimular a navegação no ambiente. Em seguida, os alunos poderiam navegar livremente pelo AVA.

Depois, lhes foi solicitado o preenchimento de um questionário (Fig. 3) contendo 14 declarações apresentadas de forma randômica relacionadas ao modelo da pesquisa com os fatores motivacionais estudados, usando uma escala Likert de 7 (sete) pontos, cujos valores variaram de "discordo totalmente"(1) a "concordo totalmente" (7). 


\begin{tabular}{|c|c|c|c|c|c|c|c|c|}
\hline \multirow[b]{2}{*}{1} & \multirow[b]{2}{*}{ Usar o AVA é divertido. } & \multicolumn{3}{|c|}{ Discordo } & \multicolumn{4}{|c|}{ Concordo } \\
\hline & & 1 & 2 & 3 & 4 & 5 & 6 & 7 \\
\hline 2 & Usando o AVA consigo aprender sobre o assunto mais rápido. & 1 & 2 & 3 & 4 & 5 & 6 & 7 \\
\hline 3 & Eu acho fácil usar o AVA para aprender sobre o assunto. & 1 & 2 & 3 & 4 & 5 & 6 & 7 \\
\hline 4 & Usar o AVA torna meu aprendizado produtivo. & 1 & 2 & 3 & 4 & 5 & 6 & 7 \\
\hline 5 & Eu não vejo o tempo passar quando estou usando o AVA. & 1 & 2 & 3 & 4 & 5 & 6 & 7 \\
\hline 6 & Dado que eu tenha acesso ao AVA, acho que o utilizarei. & 1 & 2 & 3 & 4 & 5 & 6 & 7 \\
\hline 7 & Usar o AVA torna fácil aprender sobre o assunto. & 1 & 2 & 3 & 4 & 5 & 6 & 7 \\
\hline 8 & Usar o AVA é excitante. & 1 & 2 & 3 & 4 & 5 & 6 & 7 \\
\hline 9 & Usar o AVA melhora a qualidade do meu aprendizado. & 1 & 2 & 3 & 4 & 5 & 6 & 7 \\
\hline 10 & Eu considero fácil usar o AVA. & 1 & 2 & 3 & 4 & 5 & 6 & 7 \\
\hline 11 & Usar o AVA é prazeroso. & 1 & 2 & 3 & 4 & 5 & 6 & 7 \\
\hline 12 & Eu considero o AVA útil para o meu aprendizado. & 1 & 2 & 3 & 4 & 5 & 6 & 7 \\
\hline 13 & Acho que devemos utilizar o AVA, tanto quanto possível. & 1 & 2 & 3 & 4 & 5 & 6 & 7 \\
\hline 14 & Assumindo que eu tenha acesso ao AVA, pretendo utilizá-lo. & 1 & 2 & 3 & 4 & 5 & 6 & 7 \\
\hline
\end{tabular}

Fig. 3 - Perguntas fechadas do questionário aplicado

A questão 13 foi descartada, por ter gerado interpretações que a colocaria presente em dois fatores distintos, este fato não foi identificado quando dá aplicação do piloto. Em avaliação posterior a aplicação do questionário foi percebida que esta declaração poderia ser classificada tanto como Intenção de Uso como Utilidade Percebida.

Os fatores obtidos alcançaram bom coeficiente de confiabilidade interna, alfa $(\alpha)$ de Crombach [Hair et al. 1998], conforme observado na Fig. 4.

\begin{tabular}{|lc|}
\hline Fator Motivacional & Alfa de Crombach \\
Prazer Percebido & 0,88 \\
Facilidade Percebida & 0,84 \\
Utilidade Percebida & 0,76 \\
Intenção de Uso & 0,82 \\
\hline
\end{tabular}

Fig. 4 - Alfa de Crombach

Perguntas abertas, relacionadas aos fatores motivacionais, foram colocadas para validação do experimento e um espaço para comentários dos participantes foi disponibilizado. Todo o processo foi acompanhado pelo pesquisador a fim de observar o comportamento dos participantes.

\section{RESULTADOS DO EXPERIMENTO}

Perfil demográfico dos alunos participantes do experimento:

- Faixa etária entre 14 a 16 anos de idade,

- Todos com computador e acesso a internet em casa,

- $90 \%$ utilizam a internet no celular,

- $80 \%$ utilizam a internet diariamente. 


\begin{tabular}{|ccc|}
\hline Série & Quantidade & Percentual \\
$\mathbf{1 0}$ & 9 & $45 \%$ \\
$\mathbf{2}$ ? & 11 & $55 \%$ \\
& & \\
\hline Sexo & Quantidade & Percentual \\
Masculino & 7 & $35 \%$ \\
Feminino & 13 & $65 \%$ \\
\hline
\end{tabular}

Fig. 5 - Variáveis Demográficas

Através da soma da pontuação atribuída pelos alunos a cada aspecto observado e posterior divisão pelo número de respondentes, foi obtida a média de cada aspecto (Fig. 6). De forma análoga, após somar os valores de cada aspecto referente a determinado fator e dividir esta soma pelo número de aspectos do fator foi obtida a média para cada fator observado. A média geral de 5.17 pontos em um total de 7.0 pontos foi obtida somando-se os valores médios de cada fator e dividindo esta soma pelo número de fatores avaliados no experimento, ou seja, quatro (Prazer Percebido, Facilidade Percebida, Utilidade Percebida e Intenção de Uso).

A Fig. 6 apresenta o valor médio, a partir dos valores atribuídos pelos alunos, para cada aspecto que compõe o questionário e a média obtida para cada fator da pesquisa. Os alunos participantes se mostraram motivados pela utilidade do AVA (média $=5,70$ pontos na escala Likert utilizada), o que foi confirmado pelas informações contidas nas questões abertas sobre a variável utilidade percebida.

Os participantes, em geral, declararam achar fácil usar o AVA (média $=5,59$ pontos) e o prazer percebido por eles foi o que obteve a menor média (média $=4,36$ pontos), possivelmente, devido ao momento de ocorrência do experimento, qual seja, o horário de intervalo das aulas.

\begin{tabular}{|c|c|c|c|}
\hline \multirow[t]{2}{*}{ Fator } & \multirow[t]{2}{*}{ Declaração } & \multicolumn{2}{|c|}{ Média } \\
\hline & & Declaração & Fator \\
\hline \multirow[t]{5}{*}{ Prazer Percebido } & & & 4,36 \\
\hline & Usar o AVA é divertido. & 4,85 & \\
\hline & Eu não vejo o tempo passar quando estou usando o AVA. & 4,65 & \\
\hline & Usar o AVA é excitante. & 3,40 & \\
\hline & Usar o AVA é prazeroso. & 4,55 & \\
\hline \multirow[t]{4}{*}{ Facilidade Percebida } & & & 5,59 \\
\hline & Eu acho fácil usar o AVA para aprender sobre o assunto. & 5,53 & \\
\hline & Usar o AVA torna fácil aprender sobre o assunto. & 5,55 & \\
\hline & Eu considero fácil usar o AVA. & 5,70 & \\
\hline \multirow[t]{5}{*}{ Utilidade Percebida } & & & 5,70 \\
\hline & Usando o AVA consigo aprender sobre o assunto mais rápido. & 5,37 & \\
\hline & Usar o AVA torna meu aprendizado produtivo. & 5,60 & \\
\hline & Usar o AVA melhora a qualidade do meu aprendizado. & 5,85 & \\
\hline & Eu considero o AVA útil para o meu aprendizado. & 6,00 & \\
\hline \multirow[t]{3}{*}{ Intenção de Uso } & & & 5,03 \\
\hline & Dado que eu tenha acesso ao AVA, acho que o utilizarei. & 4,85 & \\
\hline & Assumindo que eu tenha acesso ao AVA, pretendo utilizá-lo. & 5,20 & \\
\hline
\end{tabular}

\section{Fig. 6 - Valores médios obtidos}

Foi observado ao longo do experimento que apesar de alguns alunos elogiarem as diferentes formas de navegação, vários alunos tiveram dificuldade em localizar algum item em tela. Em um dos casos, uma janela de atualização do sistema operacional se 
sobrepôs na parte inferior da tela às opções de navegação do AVA, o que impossibilitou o participante de visualizar essas opções. Nenhum dos participantes teve dificuldade com o uso do computador, demonstrando uma interação tranquila com o mesmo, e grande capacidade de focar em um objetivo. O tempo médio de acesso dos participantes no experimento foi de 10 (dez) minutos, apesar de o intervalo das aulas durar 30 minutos. Percebeu-se que os participantes, na maioria, demonstraram interesse no conteúdo do AVA (apenas 5 afirmaram não ter vontade de fazer o curso). Contudo, no tempo livre para navegação, 14 alunos não demonstraram interesse em navegar o conteúdo ou em vasculhar as opções tecnológicas ofertadas.

\section{DISCUSSÃO DOS RESULTADOS E SUGESTÕES DE NOVAS PESQUISAS}

Nesta pesquisa foi identificado que alunos da instituição privada estudada têm como principal motivador para utilizar um ambiente virtual de aprendizagem o fator utilidade percebida, o que confirma pesquisas anteriores sobre a importância da utilidade percebida no uso de uma tecnologia [Dias 1998, 2001, 2005] [Dias et al. 2005] [Dias, Mariano e Vasques 2002] [Silva 2006] [Silva e Dias 2004]. Com o pequeno número de participantes do experimento (20 indivíduos) e sendo estes de uma região específica do Rio de Janeiro, esta pesquisa não pode ser considerada como conclusiva, mas sim indicativa dos fatores avaliados e permitindo o surgimento de insights sobre o fenômeno em estudo.

Apesar de Dias [Dias 2001] alertar para a importância do fator prazer percebido quando o grupo avaliado é de adolescentes, nesse experimento essa variável obteve a menor média dentre os fatores motivacionais observados. Talvez esse resultado seja devido à velocidade com que os alunos concluíram as tarefas (média de 10 minutos), e por não terem navegado pelas opções tecnológicas mais lúdicas oferecidas, tais como, bate-papo, simulador, conforme observado durante o experimento. Contudo, uma das tarefas solicitava informações biográficas e fazia com que eles, obrigatoriamente, utilizassem uma ferramenta de visualização de conteúdo. Essa ferramenta foi bastante elogiada pelos alunos em relação ao conteúdo e à forma de apresentação.

O baixo valor obtido em intenção de uso pode ser explicado, talvez, pelo pouco tempo de uso e pela falta de treinamento não propiciando que os alunos tivessem contato com todas as opções tecnológicas oferecidas como, bate-papo, fórum, vídeo, etc.

Foi observado que o ambiente e-Proinfo, algumas vezes, utiliza a parte inferior da tela para disponibilizar abas de navegação. Como essa parte da tela, às vezes, é utilizada pelo Sistema Operacional, o aluno fica impossibilitado de visualizar parte importante do conteúdo oferecido. Por exemplo, durante o experimento, o computador de um dos alunos estava solicitando a habilitação de um plug-in e essa solicitação estava sobreposta às abas de navegação, ofuscando-as. Assim, um melhor posicionamento destas opções deveria ser avaliado pela equipe de desenvolvimento do ambiente e-Proinfo.

A grande quantidade de informação a qual os participantes foram submetidos pode ter gerado uma sobrecarga cognitiva e aumentado o foco desses participantes nas tarefas definidas. Isso poderá ser validado através de uma pesquisa futura que considere outras informações como tempo de uso do AVA e treinamento prévio.

Como esses alunos são, em sua maioria, usuários diários de internet, as tecnologias oferecidas não eram novidade para eles, o que pode explicar a baixa intenção de uso. A 
fim de perceber se há aumento da intenção de uso, uma pesquisa futura poderá verificar o impacto da introdução de ferramentas de ensino inovadoras, por exemplo, as que utilizam o conceito de haptics (sensação de toque que o usuário recebe ao tocar uma superfície interativa, pode ser uma resposta sonora, visual ou tátil) [San Diego et al. 2012].

Essa pesquisa coletou informações sobre alunos da rede privada. Sugere-se que novos estudos similares contemplem alunos da rede pública a fim de comparar os resultados aqui obtidos com o uso da mesma plataforma de ensino por alunos da rede pública e verificar se há diferenças relevantes quanto ao uso dessa tecnologia.

\section{CONCLUSÕES}

Em sua pesquisa, Dias [Dias 2001] observou uma diferença significante entre adultos e não adultos quanto aos fatores motivacionais para o uso de uma tecnologia. Assim, nessa pesquisa, foi esperado que os indivíduos na faixa etária estudada fossem motivados mais pelo prazer percebido do que pelos outros fatores motivacionais avaliados.

No entanto, foi observado nesse experimento que o grande motivador foi a utilidade percebida, o que pode ser devido à capacidade dos participantes em focar um objetivo, demonstrada durante o experimento, e pelo pouco tempo disponível à livre navegação.

O fator prazer percebido obteve a menor média dentre os fatores avaliados, o que pode estar associado à velocidade de execução das tarefas e ao não aproveitamento das ferramentas lúdicas oferecidas no ambiente.

\section{REFERÊNCIAS}

CRESWELL, J. W. (2010) Projeto de Pesquisa: métodos qualitativo, quantitativo e misto. Editora ArtMed.

DAVIS, F. D. (1986) A technology acceptance model for empirically testing new end user information systems: theory and results. Tese (Doutorado). MIT, Boston, MA.

DAVIS, F. D. (1989) Perceived usefulness, perceived ease of use and user acceptance of computer technology, MIS Quarterly, Minneapolis.

DIAS, A. F. S.; SILVA, M. F.; SCHMITZ, E.; DIAS, D. S. (2005) Motivational measures of TAM factors: an investigation of handicapped users in Rio de Janeiro. In: BALAS 2005 - The Business Association of Latin American Studies, Madri, Espanha, Anais BALAS 2005.

DIAS, D. S. (1998) Managers' motivation for using information technology. Industrial Management \& Data Systems, Wembley, Inglaterra, n. 7/8, p.338-342.

DIAS, D. S. (2001) Motivation for Using Information Technology In. SZEWCZAK, E. J.; SNODGRASS, C. R. Human Factors in Information Systems. Hershey: IRMA Press, cap.5,p.55-60.

DIAS, D. S. (2005) Motivation for using microcomputers. In: Mehdi Khosrow-Pour (ed.). Encyclopedia of Information Science and Technology, v. 4, Idea Group Publishing, p. 2030-2035. 
DIAS, D. S.; MARIANO, S. R. H.; VASQUES, R. P. (2002) Antecedents of Internet use among Brazilian information systems students. Issues in Information Systems, IIS, Outubro.

Dias, D. S.; Silva, M. F. (2010); Como Escrever uma Monografia: manual de elaboração com exemplos e exercícios. São Paulo: Atlas.

FISHBEIN, M.; AJZEN, I. (1975) Belief, attitude, intention and behavior: an introduction to theory and research. Reading, MA: Addison-Wesley. Disponível em: <http://www.people.umass.edu/aizen/f\&a1975.html>. Acesso: 24 abr. 2013.

HAIR JR., J. F.; $\quad$ ANDERSON, R. E.; TATHAM, R. L.; BLACK, W. C. (1998) Multivariate Data Analysis. $5^{\text {th }}$ ed. Upper Saddle River: Prentice Hall.

MEC (2013) E-Proinfo - Ambiente Colaborativo de Aprendizagem. Disponível em: http://e-Proinfo.mec.gov.br. Acesso: 10 mar.

Silva, M. F. (2006) Fatores Humanos e sua influência na Intenção de Uso de Sistemas de Informação. Tese (Doutorado). COPPEAD/UFRJ, Rio de Janeiro, RJ.

SILVA, M. F. da; DIAS, D. de S. (2004) Intenção de Uso de Tecnologia de Informação: um estudo sobre a influência do contexto social em uma empresa do setor acadêmico brasileiro. In: XXXIX Asamblea Anual CLADEA - Consejo Latinoamericano de Escuelas, 2004, Puerto Plata. Anais del XXXIX CLADEA.

BUZATO, Marcelo E. K. (2003) Letramento digital abre portas para o conhecimento. EducaRede, 11 mar.

SAN DIEGO, J. P. et al. (2012) Researching haptics in higher education: The complexity of developing haptics virtual learning systems and evaluating its impact on students' learning. Computers \& Education v. 59, p.156-166. 